\title{
Transfer Factors or Dialyzable leukocyte Extracts as Immunomod- ulating Peptides: A Conceptual Review on Broad Spectrum of Therapeutic Areas, Immunologic and Clinical Responses, Trends and Perspectives

\author{
Dina Tulina*, Mike KS Chan, Michele WBF and Shing Yi Pan \\ Stellar Biomolecular Research, Germany
}

*Corresponding author: Dina Tulina, Stellar Biomolecular Research, 205 Klosterstrasse, 67480, Edenkoben, Germany

\begin{abstract}
Immune Modulating Peptides (IMP) discussed in literature as a universal immunocorrectors with wide areas of usage which balance the immune system without causing global immune suppression or overreaction. The present article aim is to review the effect and drawbacks of IMPs in form of Dialyzable leukocyte Extracts (DLE) or Transfer Factors (TF) during and following chemotherapy, radiotherapy, drugs interactions, cancer response and clinical outcomes in different cases of cancers such as metastatic breast cancer, glioma, prostate cancer, osteosarcoma and others. The considered cases of immune overreaction and IMP's impact include autoimmune diseases such as rheumatoid arthritis, multiple sclerosis and allergic reactions. The most difficult therapeutic decision is to choose the right treatment for patients with cancer and autoimmune diseases as they both attempt to supervise and control the immune system, managing immune cells in opposite sides. As patients with autoimmune disease experience difficulty to stay in such treatment when immune cells stimulated in order to identify and destroy cancer cells the IMP as immunocorrectors become one of the main and only healthy choices. The universal mechanism of IMPs action and the absence of contraindications extend the indications for their use in pediatric practice. This includes the complex treatment of respiratory viral infections, the possibility of using in frequently ill children with clinical signs of immune dysfunction and acute intestinal infections. According to the reports on safety, absence of adverse and side effects along with positive outcome for patients the IMPs considered as valuable immunocorrector.
\end{abstract}

\section{Keywords}

Immune modulating peptides, Immune regulation, Transfer factor, Dialyzable leukocyte extracts

\begin{abstract}
Abbreviations
IMP: Immune Modulating Peptides, TF: Transfer Factor; DLE: Dialyzable leukocyte Extracts; NK: Natural Killer Cells; IL: Interleukin; HIV: Human Immunodeficiency Virus; TNF-a - Tumor Necrosis Factor Alpha; IFN-y: Interferon Gamma IFN-g; RANTES: Regulated on Activation, Normal T Cell Expressed and Secreted; hBD-2: Human Beta Defensin 2
\end{abstract}

\section{Introduction}

Immune Modulating Peptides (IMP) are exceptional specific inhibitors of protein-protein interactions and, hence, are beneficial modulators of protein-mediated signaling of immune system. IMP's effect based on reinstating immune balance without causing global immune suppression or overreaction. Technology uses specific peptides with certain properties and abilities to stimulate an antigen-specific expansion of regulatory $T$ cells - leading mediators of immune tolerance. These cells are qualified to suppress autoantigen-specific helper $\mathrm{T}$ cells, which release proinflammatory cytokines and are reliable for immune pathology in autoimmune diseases. Transfer factors (TF) include both Inducer fractions and Regulator fractions - historically called "suppressor fractions". Inducer fractions transport an apparently mature immune response from donor to recipient. Regulator fractions help control overreactions and limit allergies and autoimmune conditions. Inducer fractions strengthen antigenic stimulus, which induces production of IFN- $\gamma$, IL- 2 and TNF- $\alpha$ by CD4+Th1 cells

Citation: Tulina D, Chan MKS, Michele WBF, Pan SY (2019) Transfer Factors or Dialyzable leukocyte Extracts as Immunomodulating Peptides: A Conceptual Review on Broad Spectrum of Therapeutic Areas, Immunologic and Clinical Responses, Trends and Perspectives. Int J Immunol Immunother 6:039. doi. org/10.23937/2378-3672/1410039

Accepted: November 02, 2019: Published: November 04, 2019

Copyright: (c) 2019 Tulina D, et al. This is an open-access article distributed under the terms of the Creative Commons Attribution License, which permits unrestricted use, distribution, and reproduction in any medium, provided the original author and source are credited. 
[1]. As result, cell-mediated immune response develops against target antigen [2], and it comprises interleukins (IL-6 and IL-8) produced by activated monocytes [3,4]. Particular factors that decrease cell mediated immunity, Th2 supremacy are age, immunodeficiency, cytotoxic cancer treatments, chronical stress, metastatic diseases, environment, etc. Suppressor fractions control and regulate immune response to an antigen and stimulating IL-10 formation and inhibitory cytokines by Th2 cells $[5,6]$. The adjuvant-like TF components have a non-specific activity expressed by enhancing immune response to other antigens or allergens [7].

The present article aim is to review the effect and drawbacks of IMPs in form of Dialyzable leukocyte Extracts (DLE) or Transfer Factors (TF) during and following chemotherapy, radiotherapy, drugs interactions, cancer response and clinical outcomes in different cases of cancers such as metastatic breast cancer, glioma, prostate cancer, osteosarcoma and others.

Current anticancer therapy tactics include surgery, radiotherapy or chemotherapy which confirmed improvement of disease prognosis and has increased survival. In patients with breast cancer, antineoplastic chemotherapy has improved overall clinical outcome. Anyway, various side effects linked with chemotherapy and radiotherapy. It is clear that side effects, not only affect tumour, but also target bone marrow activity and divide lymphocytes causing lymphocytopenia [8] which may induce subsequent clinical immunodeficiency [9]. It is confirmed that chemotherapeutic drugs lead to T-cell depletion, which is more serious in CD4+ than in CD8+ T lymphocytes, decline in dendritic cell function and an alteration in productivity of pro-inflammatory and anti-inflammatory cytokines.

Furthermore antineoplastic chemotherapy cause side effects like fatigue $[10,11]$, skeletal muscle wasting and atrophy [12], also increased degree of tumor necrosis factor, inactivity and weight loss.

Improvement of immune function defend from side effects caused by chemotherapy. Immunotherapy agent boost populations of T-cells, dendritic and natural killer (NK) cells that are main powerful effectors in host antitumor response. Immunotherapy agents considered as alternative therapy and administered to enhance antitumor immunity and to improve clinical outcome to cancer chemotherapeutic treatment.

\section{Materials and Methods}

Conceptual review was made of studies conducted on common autoimmune diseases and immune deficiencies such as cancers using IMPs. Searches included database of Ovid Medline, Science Direct, The Lancet, PubMed and Elsevier applying keywords of interested therapeutic area and IMPs.

\section{Results - Immunologic and Clinical Response}

Positive responses to TF are confirmed through various tests: Delayed hypersensitivity skin test, reaction to alloantigen, mitogen: specific and non-specific types of T cell, NK cell activity, cytokines activity [2]. TF in osteosarcoma cases increased cell mediated cytotoxicity [13] and in Varicella cases with acute leukaemia in children and Herpes simplex virus improved T cell function [14-16]. Elevated C3 level restored to normal, no new infection, absence of eczema was observed in Wiskott - Aldrich syndrome [17]. Prostate cancer patients using TF during treatment showed higher survival rates among same group [18] and in HIV cases increased levels of Th cells and cytotoxic T cells [19]. Lung cancer patients using TF demonstrated longer survival rate [20]. TF in Hepatitis $C$ cases stimulated Th1, which helps in viral particles clearing $[21,22]$. Administration directly to experimental glioma reduced tumor size and increased CD2+, CD4+, CD8+ and NK cell counts with increased apoptotic tumor cells percentage and tumor tissue expressing Th1 cytokines. Researchers observed additive antitumor effect of TF combined with chemotherapy [23].

Decrease of inflammatory infiltrates, morphological changes to similar of normal prostate, and prostatein decreased to basal levels was reported in DLE group with decreased CD45 expression and proinflammatory cytokines TNF- $\alpha$, IFN- $\gamma$, IL-6, IL-17. In summary: DLE is able to modulate inflammatory response in experimental autoimmune prostatitis [24].

Prostate cancer is $2^{\text {nd }}$ frequently diagnosed cancer in men worldwide. In vivo, DLEs reduced metastatic dissemination and inhibited tumour growth. DLEs antineoplastic effect correlated with changes in tumour infiltration, increased serum concentrations of IL-12 and CXCL1, reduced levels of vascular endothelial growth factor. Results: Antineoplastic impactassociated with immunomodulatory effect, not by direct effect on cancer cells, which indicate DLEs as beneficial adjuvant therapy in prostate cancer patients [25].

Breast cancer patients with DLE as adjuvant achieved metastatic lesions regression faster than control group. Subjects with metastatic breast cancer without DLE had persistent thyroid lesions and new lesion around aorta following 2 years of chemotherapy in contrast to group with retroperitoneal retrohepatic metastases with partial regression after 4 months of DLE treatment and 5 chemotherapy cycles [26]. TF as chemotherapy adjuvant associated with tumor regression and temporary cancer stabilization or reduced demand for other treatment modalities and have meaning as adjuvant therapy in certain malignancies [27].

Stage D3 prostate cancer survival rate on conventional therapy is very poor. During 1-9 years follow-up 
complete remission obtained in $4 \%$, partial remission in $12 \%$ and no metastasis progression in $28 \%$ patients. Median survival was 126 weeks, higher than survival rates reported in literature for same stage patients [18].

Osteosarcoma is malignant bone tumor with intention to metastasis and immune response loss of cytotoxic cells types. Assessment after surgery, neoadjuvant chemotherapy and specific TF showed that $63 \%$ patients increased T lymphocytes number, 75\% increased cytotoxic T lymphocytes and $87.5 \%$ patients returned to phase I of Levins classification [28].

Disseminated renal cell carcinoma treated with TF as immune stimulant showed temporary metastases stabilization while patients without clinically evident metastases with high risk for recurrent disease remained disease free [29].

Nasopharyngeal carcinoma patient's refractory to conventional therapy treated with TF slowed tumor growth and associated with intense lymphocytic tumor infiltration and reconstitution of delayed cutaneous hypersensitivity reactions to microbial recall antigens which suggest favorable response to TF immunotherapy [30].

Myelosuppression is chemotherapy side effect along with lymphopenia, neutropenia and thrombocytopenia [9]. DLE protective effect demonstrated on NK cells together with CD4+ T, CD8+ T and CD19+ B lymphocytes. No myelosuppression in lymphoid populations observed in patients receiving DLE while without DLE absolute numbers of CD4+, CD8+ and $B$ lymphocytes reduced. NK cells and B lymphocytes increased 1 month after chemotherapy with DLE as adjuvant. IL-3 levels dropped by $80 \%$ in control group without DLE compare to decrease by $34 \%$ only with DLE. IL-7 levels elevated with DLE by $11 \%$ decreased by $30 \%$ in control group [26].

The best outcome of neoplastic disease management are obtained by surgery in addition with chemotherapy and radiotherapy. All procedures influence immune system which often disturbed due to low leukocytes number, lymphocytes and side effects resulting from depressed immuninty. Immunostimulatory agents are helping to escape or minimize harmful effects like TF was used in cancer therapy by Fudenberg [31] and by other researchers in non neoplastic cases [31-33]. Regarding total leukocyte count, 65\% patients presented 6-month increase in values and $83.3 \%$ in 12 months with leukocytes amount grow ranged from $1.9 \%$ to $103 \%$ and from $2.1 \%$ to $170 \%$ respectively. Total lymphocytes growth occured in $66.7 \%$ patients in 6 months and in $80 \%$ cases in 12 months. This elevation extended from $1.5 \%$ to $85 \%$ in 6 months and from $0.2 \%$ to $137.7 \%$ in 12 months. Statistical survey of these range was very significant [34].
Cancer and autoimmunity have general background, both attempt to supervise pulling immunity in opposite ways. Cancer and autoimmune diseases increase risks and treatments outcome and patients experience difficulty to stay in treatment protocol.

Autoimmune disorders cause inflammation which can lead to cancer. For example colitis or inflammatory bowel disease can rise chance of colorectal cancer. Chronic inflammation harm cell's DNA and may result in unlimited cell growth. In order to reduce inflammation immunosuppressors are frequently adviced and both assumed on increasing multiple cancers risk. Arthritis and psoriasis associated with increased lymphoma risk due to their pathophysiology, treatments or combination of these factors [35]. Autoimmune diseases treatment is complicated by risk of activating pathological process which possible to avoid with modern immunomodulatory therapy. With IPMs appearance there is hope for positive treatment outcome. Long-term therapy effect with IMP in $70 \%$ rheumatoid arthritis casesreported excellent, very good and good results. Among those $34.3 \%$ responded good, $37.1 \%$ showed very good outcome and continued treatment with nonsteroid products and IMP. 28.6\% with stage 1 rheumathoid arthritis achieved excellent results and withdrawn nonsteroid therapy. Hence, TF adjuvant therapy may be considered benefitial for patients with rheumatoid arthritis [36].

Immunomodulation therapy may promise significant benefitsin management of dermatological autoimmune and inflammatory conditions [37,38]. Clinical data suggests that DLE in a form of STF may be used as a monotherapy or in combination with other immunotherapeutic modalities in management of eczema and other forms of dermatitis.

The 2 year, double blind, controlled TF trial in multiple sclerosis treatment showed drop on disability progression with remarkable outcome after 18 months from its commencement. Patients who received and continued TF demonstrated slower rate of multiple sclerosis progression compare to placebo group with significantly faster progression rate during trial, which slowed with TF treatment commencement. In addition, 470 patients with clinically definite multiple sclerosis are being treated in TF open study. Disease progression rate appears to be similar in patients who administered TF in initial trial. Follow-up study of 1980 TF trial patients and open study of 470 patients confirmed: TF has positive effect on slowing course of multiple sclerosis [39].

Although various mechanisms involving antibodies and different cell types take apart, Th1 and Th2 cells imbalance appears to perform one of main role in allergy formation. Another subpopulations: Th17, CD4 FOXP3 and Th9 positive regulatory $T$ lymphocytes 
also engaged in allergic response. TF induces mRNA expression of IFN-g, osteopontin, RANTES, and hBD2 in human healthy subjects. TF was administered in different immune dysfunctions like allergies, immunodeficiencies, infectious diseases and tumors. TF recipients along with their conventional treatment frequently have better clinical evolution than without it, thus reach prompt and better resolution of allergic response [40].

\section{Discussion}

IMP was well studied for more than half century. $\mathrm{TF}$ is potent to improve existing cell-mediated immunity responses also transfer information to identify and stimulate new response to various pathogens. Thereby it has been considered as essential tool, not only for treating, but also for preventing pathologies induced by them [31]. TF's pleiotropic properties shouldn't be overlooked, as lymphocyte extract comprises of fractions with both enhancer and suppressor features, carrying several antigenic specificities [5]. TF function in patients with virally induced cancers by increasing their potency to destroy respective virus and/or by increasing their capacity to recognize and eliminate novel formed cancer cells. Simultaneously, by stimulating suppressor cell functions, it promotes control of subsequent inflammatory processes [41].

Beneficial fact is that TF lack of viable cells which play role in graft versus host reaction, thus it is not immunogenic, contain no histocompatibility antigens. To specify target TF influence expression of antigen receptors on cells. Increased Th1 in turn repress production of Th2 and its cytokines like IL-4, IL-5, IL-6, and IL-13. TF promotes immune response mediated by cytokines that indirectly induce proliferation of hematopoietic progenitor cells in bone marrow [42]. DLE generally known as TF, is immunotherapy agent that demonstrated ability to improve immunological response in cancer patients $[43,44]$. The studies with DLE as adjuvant therapy have continually reported improvement in clinical response to treatment [4547].

TF has important immune modulatory ability and lowers immunity to normal level in overreactions besides stimulates immune response, supports maturation and thymocytes differentiation, restores peripheral lymphocytesfunction, recovers humoral immunity via B lymphocytes differentiation, develops plasmocytes and synthesizes specific humoral antibodies, activates $\mathrm{T}$ lymphocytes and lymphokine production also increases activity of mononuclear phagocytic system [34]. Researchers confirmed that in control groups after several chemotherapy cycles quantity of lymphocytes decreased to below reference range. Contrary, in patients with DLE as adjuvant, $\mathrm{CD} 4+$ and $\mathrm{CD} 8+$ and B lymphocytes stayed within median value with increased NK cells amount after chemotherapy. It was concluded in many studies that DLE treatment supports considerably immunological recovery after numerous chemotherapy, ameliorate treatment compliance and quality of life during chemotherapy.

Therefore, TF gives attractive option to complement chemotherapy, which might strengthen immune system after disturbances following chemotherapy side effects $[3,48,49]$. Main TF benefit as immunotherapeutic agents is possibility to stimulate rapid immune reaction against pathogen within 24 hours and reducetime for patient immune response to 9-13 days [50]. Combination of results from different TF studies in one and extracting treatment outcomes of cancer with chemotherapy, autoimmune disorders, allergies also immunodefficiencies and infection diseases can be used by clinicians for decision-making and improving patient's life quality. Multiple studies showed TF as improving clinical and immunological response, symptomatology asabsolute lymphocytes numbers were always higher than expected as compared to control groups. TF doubtlessly accentuate immunological protection provided by DLE during chemotherapy. Median survival rates after metastases appearance is approximately 20-25 months and as time is limited it is higly important to obtain clinical response as fast as possible. It was observed that those metastatic patients receiving DLE demonstrated improved clinical responses within 6-12 months [26].

Cancer cases are more serious due to tumor itself in addition to chemotherapy or radiotherapy and corticosteroids, also affects immune system, contributing to immunosuppression accentuation. Different immunomodulators applied to reverse situation in purpose not only of improving immune response, minimizing chemotherapy side effects and radiotherapy, but of preventing protocols used to be interrupted, which compromises treatment results [51,52]. Lymphocytes with $\mathrm{T}$ subclasses are fundamental for immune reaction, particularly with respect to solid tumors. Thus, combats to this tumor type have objective of making T-lymphocytes active and competent $[53,54]$. It is obvious that TF stimulate leukocytes activation, total lymphocytes and their subclasses, leading to immune response induction [34].

TF can display multiple regulatory effects on individual aspects of immune system due to stimulation of expression of transcription factor retinoic acid receptor-related orphan receptor-gamma-t (RORyt) and enhances proportion of CD4+ RORyt+ cells, whi$c h$ in turn leads to stimulated expression of gene for IL-17 and increased IL-17production. As such main effect confirmed in pathway of Th17 cell development and IL-17 production [55]. IL-17 is highly pleiotropic cytokine having multiple effects in various immuno- 
logical situations, including inflammation, autoimmunity, transplantation reactions, asthma and anti-tumour immunity [56,57], enhance local inflammatory reactions and modulate immune system functions $[58,59]$. This pathway may represent main mechanism of immunomodulatory and immunotherapeutic TF actions described in various models [60]. According to last half century studies DLE or TF determines wide range of possibilities to use but due to TF is complex group of many low molecular weight proteins (more than 200), its precise molecular structure and mechanisms of action have not been elucidated yet, which means different studies might be using different TFs and as such need more comparable research conducted. However its use in clinical practice as IMP is absolutely valuable but before administration it is important to evaluate specificity, potency, and best dose, trying to individualize treatment for each patient [61].

At present time, prediction and prevention positioned as pillars of medicine. TF is prospective candidate for prevention and treatment of existing and novel emerging pathogens including their clinical appearance. Inflammation, contrariwise, must also be managed by suppressing infections and also by direct impact on its complex mechanisms by inducing power of suppressor lymphocytes and controlling cytokine secretion. TF's significant advantages are: efficiency on treating and preventing infections, reasonable manufacturing price, safety and absence of toxicity. Indeed, TF are universal immunocorrectors with proven multiple studies effects.

\section{Conflict of Interest}

There are no conflicts of interest to declare.

\section{References}

1. Arnaudov A, Kostova Zh (2015) Dialysable leukocyte extracts in immunotherapy. Biotechnology and Biotechnological Equipment 29: 1017-1023.

2. Krishnaveni M (2013) A review on transfer factor an immune modulator. Drug Invent Today 5: 153-156.

3. Ojeda MO, Van't Veer C, Fernandez-Ortega CB, Arana Rosainz Mde J, Buurman WA (2005) Dialyzable leukocyte extract differentially regulates the production of TNFa, IL-6, and IL-8 in bacterial component-activated leukocytes and endothelial cells. Inflamm Res 54: 74-81.

4. Robles-Contreras A, Vizuet L, Rivera E, Serafin-Lopez J, Estrada-Garcia I, et al. (2011) Down regulation of IL-8 and IL-6 in human limbal epithelial cells cultured with human dialyzable leukocyte extracts. Rev Alerg Mex 58: 147-154.

5. Lawrence HS, Borkowsky W (1996) Transfer factor - current status and future properties. Biotherapy 9: 1-5.

6. Burger DR, Vandenbark AA, Daves D, Anderson WA Jr, Vetto RM, et al. (1976) Nicotinamide: Suppression of lymphocyte transformation with a component identified in human transfer factor. J Immunol 117: 797-801.

7. Gottlieb AA, Maziarz GA, Tamaki N, Sutcliffe SB (1980) The effects of dialyzable products from human leukocyte extracts on cutaneous delayed-hypersensitivity response. $J$
Immunol 124: 885-892.

8. Mackall CL, Fleisher TA, Brown MR, Magrath IT, Shad AT, et al. (1994) Lymphocyte depletion during treatment with intensive chemotherapy for cancer. Blood 84: 2221-2228.

9. Van der Most RG, Currie AJ, Robinson BWS, Lake RA (2008) Decoding dangerous death: How cytotoxic chemotherapy invokes inflammation, immunity or nothing at all. Cell Death Differ 15: 13-20.

10. Piper BF, Borneman T, Sun VC, Koczywas M, Uman G, et al. (2008) Cancer-related fatigue: Role of oncology nurses in translating national comprehensive cancer network assessment guidelines into practice. Clin $\mathrm{J}$ Oncol Nurs 12: 37-47.

11. Berger AM (1998) Patterns of fatigue and activity and rest during adjuvant breast cancer chemotherapy. Oncol Nurs Forum 25: 51-62.

12. Goodman MN (1991) Tumor necrosis factor induces skeletal muscle protein breakdown in rats. Am J Physiol 260: E727-E730.

13. Fudenberg $\mathrm{HH}$ (1976) Dialyzable transfer factor in treatment of human osteosarcoma: An analytic review. Ann N Y Acad Sci 277: 545-557.

14. Steele RW, Myers MG, Vincent MM (1980) Transfer factor for the prevention of Varicella zoster in childhood leukemia. N Eng J Med 303: 355-359.

15. Russell W Steele, Heberling RL, Eichberg JW, Kalter SS, Eller JJ, et al. (1976) Prevention of Herpes simplex virus Type 1 fatal dissemination in primates with human transfer factor. Transfer Factor: Basic Properties and Clinical Applications. New York: Academic Press 381-386.

16. Viza D, Vich JM, Phillips J, Rosenfeld F, Davies DA (1986) Specific transfer factor protects mice against lethal challenge with Herpes simplex virus. Cell 100: 555-562.

17. Levin AS, Spitler LE, Stites DP, Fudenberg HH (1970) Wiskott - Aldrich syndrome, a genetically determined cellular immunologic deficiency: Clinical and laboratory responses to therapy with transfer factor. Proc Natl Acad Sci U S A 67: 821-828

18. Pizza G, De Vinci C, Cuzzocrea D, Menniti D, Aiello E, et al. (1996) A preliminary report on the use of transfer factor for treating stage D3 hormone-unresponsive metastatic prostate cancer. Biotherapy 9: 123-132.

19. Granitov NV, Karbysheva NV, Sultanov LV, Mack Cosland K, Oganova E (2002) Use of activated transfer factor in treatment of HIV-infected patients. Russ J HIVIAIDS Relat Probl 6: 79-80.

20. Pilotti V, Mastrorilli M, Pizza G, De Vinci C, Busutti L, et al. (1996) Transfer factor as an adjuvant to non-small cell lung cancer therapy. Biotherapy 9: 117-121.

21. Milich DR, Chen MK, Hughes JL, Jones JE (1998) The secreted hepatitis precove antigen can modulate the immune response to the nucleocapsid: A mechanism for persistence. J Immunol 160: 2013-2021.

22. Tsai SL, Liaw TF, Chen MH, Huang CY, Kuo GC (1997) Detection of type-2 like $T$ helper cells in hepatitis $C$ detection: implications for hepatitis C virus chronicity. Hepatology 25: 449-458.

23. Pineda B, Estrada-Parra S, Pedraza-Medina B, Rodriguez-Ropon A, Perez R, et al. (2005) Interstitial transfer factor as adjuvant immunotherapy for experimental glioma. J Exp Clin Cancer Res 24: 575-583.

24. Perez-Alvarado C, Gomez C, Reyes M, Garcia M, Perez E, et al. (2017) Anti-inflammatory effect of dialyzable leukocyte extract in autoimmune prostatitis: Evaluation in animal model. Biomed Res Int. 
25. Hernandez-Esquivel MA, Perez-Torres A, Romero-Romero L, Reyes-Matute A, Loaiza B, et al. (2018) The dialyzable leukocyte extract TransferonTM inhibits tumor growth and brain metastasis in a murine model of prostate cancer. Biomed Pharmacother 101: 938-944.

26. Lara HH, Turrent LI, Garza-Trevino EN, Tamez-Guerra R, Rodriguez-Padilla C (2010) Clinical and immunological assessment in breast cancer patients receiving anticancer therapy and bovine dialyzable leukocyte extract as an adjuvant. Exp Ther Med 1: 425-431.

27. Meier CR, LoBuglio AF (1977) Transfer factor: A potential agent for immunotherapy of cancer. World J Surg 1: 617623.

28. Azucena Rodriguez-Flores, Paola Castillo-Juarez, Dotor Vazquez, R Gonzalez-Guzman, G Rico-Martinez, et al. (2009) Immunological evaluation of patients with osteosarcoma received adjuvant treatment with specific transfer factor. J Immunol 182: 63.

29. Montie JE, Bukowski RM, Deodhar SH, Hewlett JS, Stewart $\mathrm{BH}$, et al. (1977) Immunotherapy of disseminated renal cell carcinoma with transfer factor. J Urol 117: 553-556.

30. Goldenberg GJ, Brandes LJ (1976) In vivo and in vitro studies of immunotherapy of nasopharyngeal carcinoma with transfer factor. Cancer Res 36: 720-723.

31. Levine PH, Pizza G, Ajmera K, De Vinci C, Viza D (2011) Transfer factor in virus-associated malignancies: An underestimated weapon in prevention and treatment of cancer. Adv Tumor Virol 2: 7-20.

32. Gibson J, Basten A, Van Der Brink C (1983) Clinical use of transfer factor 25 years. Immunol Allergy Clin N A 3: 331357.

33. Kirkpatrick CH (1980) Therapeutic potential of transfer factor. N Engl J Med 303: 390-391.

34. Garritano CRO, Nubila FD, Couto RM, Fiorelli RKA, Aun LB (2017) Use of transfer factor in immunosuppressed surgical patients. Rev Col Bras Cir 44: 452-456.

35. Gelfand JM, Shin DB, Neimann AL, Wang X, Margolis DJ, et al. (2006) The risk of lymphoma in patients with psoriasis. J Invest Dermatol 126: 2194-2201.

36. Georgescu C (1985) Effect of long-term therapy with transfer factor in rheumatoid arthritis. Med Interne 23: 135-140.

37. Klokol D, Pan SY, Chan MKS, Wong MBF, Chernykh V, et al. (2018) Management of eczema with active specific immunotherapy and super transfer factor: A case report. Insights Enzyme Res 4: 1.

38. Chan MKS, Klokol D (2019) A comprehensive guide to biological medicine and wellness. Troubador, UK.

39. Frith JA, McLeod JG, Basten A, Pollard JD, Hammond SR, et al. (1986) Transfer factor as a therapy for multiple sclerosis: a follow-up study. Clin Exp Neurol 22: 149-154.

40. Gomez Vera J, Chavez Sanchez R, Flores Sandoval G, Orea Solano M, Lopez Tiro JJ, et al. (2010) Transfer factor and allergy. Rev Alerg Mex 57: 208-214.

41. Viza D, Fudenberg HH, Palareti A, Ablashi D, De Vinci C, et al. (2013) Transfer factor: An overlooked potential for the prevention and treatment of infectious diseases. Folia Biologica (Praha) 59: 53-67.

42. Vacek A, Hofer M, Schneiderova H, Svoboda J (2005) UItrafiltered pig leukocyte extract (UPLE, IMUNOR) potentiates hematopoiesis-stimulating effects of G-CSF in vitro and improves the outcome of treatment of hematopoietic radiation damage in mice with G-CSF. J Immunopharmacol Immunotoxicol 27: 647-659.

43. Rodriguez-Padilla C, Garcia FA (2005) Intra-arterial chemo inmuno target therapy plus conformal XRT in brain tumors. 16th International Congress on Anti-Cancer Treatment Paris, France.

44. Franco-Molina MA, Mendoza-Gamboa E, Zapata-Benavides $P$, Vera-Garcia ME, Castillo-Tello P, et al. (2008) Immunepotent CRP (bovine dialyzable leukocyte extract) adjuvant immunotherapy: A phase I study in non-small cell lung cancer patients. Cytotherapy 10: 490-496.

45. Fujisawa T (1985) Transfer factor immunotherapy as an adjunct to surgery in lung cancer. Nihon Kyobu Shikkan Gakkai Zasshi 23: 68-73.

46. Krown SE, Pinsky CM, Hirshaut Y, Hansen JA, Oettgen HF (1978) Effects of transfer factor in patients with advanced cancer. Isr J Med Sci 14: 1026-1038.

47. Vacek A, Hofer M, Hola J, Weiterova L, Streitova D, et al. (2007) The role of G-CSF and IL-6 in the granulopoiesis-stimulating activity of murine blood serum induced by perorally administered ultrafiltered pig leukocyte extract, IMUNOR. Int Immunopharmacol 7: 656-661.

48. Atzpodien J, Kuchler T, Wandert T, Reitz M (2003) Rapid deterioration in quality of life during interleukin-2- and alpha-interferon-based home therapy of renal cell carcinoma is associated with a good outcome. $\mathrm{Br} \mathrm{J}$ Cancer 89: 50-54.

49. Kirkpatrick CH, Rozzo SJ, Mascali JJ, Merryman CF (1985) Murine transfer factor. II. Transfer of delayed hypersensitivity to synthetic antigens. J Immunol 134: 1723-1727.

50. Arnaudov A (2017) Immunotherapy with dialyzable leukocyte extracts containing transfer factor. immunotherapy myths, reality, ideas, future.

51. Garritano CRO, Gomes JCG, Pimenteira CAP (2010) ß1-3 Glucan no tratamento do cancer de intestino. J Bras Med 98: 22-24.

52. Souza CA, Vigorito AC, Aranha FJP, Oliveira GB, Eid KAB, et al. (2000) Terapeutica citoprotetora em pacientes tratados com quimio e/ou radioterapia anti neoplasica. Rev Bras Hematol Hemoter 22: 123-128.

53. Rosenberg SA, Restifo NP, Yang JC, Morgan RA, Dudley ME (2008) Adoptive cell transfer: A clinical path to effective cancer immunotherapy. Nat Rev Cancer 8: 299-308.

54. Guinn BA, Kasahara N, Farzaneh F, Habib NA, Norris JS, et al. (2007) Recent advances and current challenges in tumor immunology and immunotherapy. Mol Ther 15: 10651071.

55. Zajicova A, Javorkova E, Trosan P, Chudickova M, Krulova $\mathrm{M}$, et al. (2014) A low-molecular-weight dialysable leukocyte extract selectively enhances development of CD4+ RORyt+ T Cells and IL-17 production. Folia Biologica (Praha) 60: 253-260.

56. Aggarwal S, Gurney AL (2002) IL-17: Prototype member of an emerging cytokine family. J Leukoc Biol 71: 1-8.

57. Kolls JK, Linden A (2004) Interleukin-17 family members and inflammation. Immunity 21: 467-476.

58. Kawaguchi M, Kokubu F, Kuga H, Matsukura S, Hoshino $H$, et al. (2001) Modulation of bronchial epithelial cells by IL-17. J Allergy Clin Immunol 108: 804-809.

59. Jones CE, Chan K (2002) Interleukin-17 stimulates the expression of interleukin-8, growth-related oncogene- $\alpha$, and granulocyte-colony-stimulating factor by human airway epithelial cells. Am J Respir Cell Mol Biol 26: 748-753.

60. Williams ME, Kauffman CA (1980) Transfer factor: A murine model. Infect Immun 27: 187-191.

61. Fabre RA, Perez TM, Aguilar LD, Rangel MJ, Estrada-Garcia I, et al. (2004) Transfer factors as immunotherapy and supplement of chemotherapy in experimental pulmonary tuberculosis. Clin Exp Immunol 136: 215-223. 